\title{
Universal equilibrium range of turbulence
}

\author{
J. Qian ${ }^{a)}$ \\ Institute of Mechanics, Chinese Academy of Sciences, Beijing, The People's Republic of China and \\ Department of Mechanical Engineering, The City College of the City University of New York, New York 10031
}

(Received 12 October 1983; accepted 7 February 1984)

The variational approach to the closure problem of turbulence theory, reported in an earlier paper, is applied to the study of the universal equilibrium range of turbulence. Two integral equations are derived for two unknown functions, taking account of viscous dissipation. The equation-error method of parameter estimation of control theory is used to obtain the approximate solution of the two integral equations. The resulting energy spectrum for the universal equilibrium range is $E(k)=\epsilon^{2 / 3} k^{-5 / 3} F\left(k / k_{d}\right)$ with $F(x)=1.19\left(1+5.3 x^{2 / 3}\right)$ $\times \exp \left(-5.4 x^{4 / 3}\right)$. Here $\epsilon$ is the energy dissipation rate and $k_{d}$ is the Kolmogorov wavenumber. The corresponding one-dimensional energy spectrum and dissipation spectrum are calculated and are in agreement with the experimental results. Since $F(x)$ is not a monotonically decreasing function, but has a maximum near $x=0.1$, the usual experimental values of the Kolmogorov constant will be greater than its theoretical value $F(0) \simeq 1.2$ and will depend upon the range of $k / k_{d}$.

\section{INTRODUCTION}

The Kolmogorov hypothesis ${ }^{1}$ asserts that for fully developed turbulence its small scales of motion have a behavior independent of the detail of the large energy-containing scales and are statistically steady and isotropic. In the smali scale range (the universal equilibrium range) the turbulence is uniquely determined by two parameters: the kinematic viscosity $\nu$ and the energy dissipation rate $\epsilon$. By dimensional analysis the energy spectrum for the universal equilibrium range must have the following form, ${ }^{2,3}$

$$
E(k)=\epsilon^{2 / 3} k^{-5 / 3} F\left(k / k_{d}\right) .
$$

Here $k_{d}=\left(\epsilon / v^{3}\right)^{1 / 4}$ is the Kolmogorov wavenumber and $F(x)$ is a dimensionless function of universal nature. In the inertial subrange, $k / k_{d} \simeq 0$, so (1) becomes

$$
E(k)=F(0) \epsilon^{2 / 3} k^{-5 / 3},
$$

which is the Kolmogorov law; $F(0)$ is the Kolmogorov constant.

Much effort has been directed to the determination of the form of $F(x){ }^{2,3}$ Historically, Heisenberg ${ }^{4}$ proposed that the action of the small eddies is equivalent to an eddy viscosity and obtained a $k^{-7}$ energy spectrum for the dissipation subrange. By assuming that the energy transfer function is proportional to the energy spectrum and that the proportionality coefficient depends on $\epsilon$ and $k$ only, $\mathrm{Pao}^{5}$ obtained

$$
F(x)=F(0) \exp \left[-1.5 F(0) x^{4 / 3}\right],
$$

and then adjusted the $F(0)$ to get the best agreement with the experimental data. Hence Pao's formula (3) is of semi-empirical nature.

Our goal in this paper is twofold. First of all it attempts to determine the form of $F(x)$ by the method of statistical mechanics without the introduction of any parameter of semi-empirical nature. Second, the validity of the variational approach to the closure problem proposed in an earlier pa-

\footnotetext{
a) Present address: P. O. Box 3928, Beijing, The People's Republic of China.
}

per $^{6}$ is to be tested again by applying it to the study of the universal equilibrium range of turbulence.

\section{AVERAGE MODAL INTENSITY AND TRIPLE CORRELATION}

In an earlier paper ${ }^{1}$ the variational approach is proposed to solve the closure problem of turbulence theory; two integral equations (the energy equation and the $\eta$ equation) are derived for two unknown functions (the energy spectrum $q(k)$ and the dynamic damping coefficient $\eta)$. During the derivation of the two integral equations it is assumed that the viscous dissipation is negligible, so they are not valid for the dissipation subrange. In order to study the universal equilibrium range the theory has to be extended to take account of viscous dissipation.

The probability distribution of a turbulent flow in its modal phase space is ${ }^{6}$

$$
\begin{aligned}
P= & \left(1-\sum_{i}\left(v_{i}-v_{i}^{\prime}\right) \frac{X_{i}^{2}-\phi_{i}}{2 \eta_{i} \phi_{i}}+\sum_{i j m} \frac{A_{i j m} X_{i} X_{j} X_{m}}{\left.\phi_{i}+\eta_{i}+\eta_{m}\right)}\right) \\
& \times \prod_{i}\left(2 \pi \phi_{i}\right)^{-1 / 2} \exp \left[-X_{i}^{2} /\left(2 \phi_{i}\right)\right] .
\end{aligned}
$$

The notation used in this paper is the same as in Ref. 6. By (4) the average modal intensity is

$$
\begin{aligned}
& \left\langle X_{i}^{2}\right\rangle=\int X_{i}^{2} P \prod_{n} d X_{n}=e_{i} \phi_{i}, \\
& e_{i}=1-\left(v_{i}-v_{i}^{\prime}\right) / \eta_{i} .
\end{aligned}
$$

In the universal equilibrium range, $v_{i}^{\prime}=0$, so

$$
e_{i}=1-v_{i} / \eta_{i}=\zeta_{i} / \eta_{i}
$$

For isotropic turbulence, $\left\langle X_{i}^{2}\right\rangle, e_{i}, \xi_{i}$, and $\eta_{i}$ are functions of $k$ only, and

$$
\begin{aligned}
& e(k)=1-v k^{2} / \eta(k), \\
& \eta(k)=\zeta(k)+v k^{2} .
\end{aligned}
$$

From (4) and (5) the triple correlation 


$$
\begin{aligned}
& \left\langle X_{i} X_{j} X_{m}\right\rangle=\int X_{i} X_{j} X_{m} P \prod_{n} d X_{n}=\frac{2 B_{i j m}}{\left(\eta_{i}+\eta_{j}+\eta_{m}\right)}, \\
& B_{i j m}=A_{i j m} \phi_{j} \phi_{m}+A_{j m i} \phi_{m} \phi_{i}+A_{m i j} \phi_{i} \phi_{j} .
\end{aligned}
$$

For the inertial subrange, $v_{i}=0$, so $e_{i}=1$, then (5) and (10) become the corresponding Eqs. (26) and (30) of Ref. 6, respectively.

\section{ENERGY EQUATION}

From the Liouville equation it follows that ${ }^{6}$

$\frac{d}{d t}\left\langle X_{i}^{2}\right\rangle=-2\left(v_{i}-v_{i}^{\prime}\right)\left\langle X_{i}^{2}\right\rangle+2 \sum_{j, m} A_{i j m}\left\langle X_{i} X_{j} X_{m}\right\rangle$.

For the universal equilibrium range $v_{i}^{\prime}=0$, then by using (10) we have

$$
\left(\frac{d}{d t}+2 v_{i}\right)\left\langle X_{i}^{2}\right\rangle=4 \sum_{j, m} \frac{A_{i j m} B_{i j m}}{\eta_{i}+\eta_{j}+\eta_{m}} .
$$

Similar to the derivation of the energy equation of Ref. 6 , from (5), (11), and (13) we obtain the following energy equation for the universal equilibrium range:

$$
\begin{aligned}
\left(\frac{d}{d t}+2 v k^{2}\right) E(k)=T(k), & \\
T(k)= & 16 \pi^{2} \int^{\Delta} \int d p d r k^{3} p r \\
& \times \frac{b(k, p, r) \tilde{q}(r)[\tilde{q}(p)-\tilde{q}(k)]}{\eta(k)+\eta(p)+\eta(r)} .
\end{aligned}
$$

Here,

$$
E(k)=4 \pi k^{2} q(k)=4 \pi k^{2} e(k) \tilde{q}(k)
$$

is the three-dimensional energy spectrum. The $b(k, p, r)$ is the geometrical factor defined in Ref. 6. In the inertial subrange $e(k)=1$, so (15) becomes the corresponding (energy) equation (39) of Ref. 6.

For stationary turbulence, Eq. (14) becomes

$$
2 v k^{2} E(k)=T(k),
$$

and $T(k)$ is still given by $(15)$.

\section{IV. $\eta$ EQUATION}

According to the variational approach, ${ }^{6}$ the $\eta$ equation is obtained by minimizing

$$
\sum_{i}\left\langle\left(\sum_{j, m} A_{i j m} X_{j} X_{m}-\left(-\zeta_{i} X_{i}\right)\right)^{2}\right\rangle
$$

with respect to the variation of $\eta$ or $\zeta$, while the average modal intensity $\left\langle X_{i}^{2}\right\rangle$ is considered a constant. By using (7)(11) this minimization leads to the following $\eta$ equation for the universal equilibrium range:

$$
\eta(k)=G(k),
$$

with

$$
\begin{aligned}
G(k)= & \nu k^{2}+\left(\frac{2 \pi}{q(k)}\right) \int^{\Delta} \int d p d r \frac{k p r}{[\eta(k, p, r)]^{2}} \\
& \times(b(k, p, r) d(r, p, k)[\eta(k, p, r)-\zeta(k)+\zeta(p)]
\end{aligned}
$$

Here

$$
\begin{aligned}
& +b^{*}(k, p, r) d(k, p, r) \zeta(p)+\frac{v k^{2} \eta(k, p, r)}{[\zeta(k)]^{2}} \\
& \times q(k) \tilde{q}(r)\{b(k, p, r)[\zeta(p)-\zeta(k)] \\
& \left.\left.+b^{*}(k, p, r)[\zeta(p)-\zeta(r)]\right\}\right) .
\end{aligned}
$$

and

$$
\eta(k, p, r)=\eta(k)+\eta(p)+\eta(r),
$$

$$
d(k, p, r)=\tilde{q}(k)[\tilde{q}(r)-\tilde{q}(p)] .
$$

The $b(k, p, r)$ and $b^{*}(k, p, r)$ are geometrical factors defined by (36) of Ref. 6. In the inertial subrange $v k^{2}=0, \eta(k)=\zeta(k)$, and $e(k)=1$, so $(18)$ becomes the corresponding equation (35) of Ref. 6.

\section{EQUATION-ERROR METHOD OF PARAMETER ESTIMATION}

The energy equation and the $\eta$ equation constitute a closed set of equations for two unknown functions $\eta(k)$ and $q(k)$ [or $E(k)]$. They are valid for the dissipation subrange as well as the inertial subrange. In the inertial subrange there is no viscous dissipation; the two integral equations are reduced to the corresponding equations of Ref. 6 and have been solved to get the following expressions for $\eta(k)$ and $E(k)$ :

$$
\eta(k)=\zeta(k)=0.268 \epsilon^{1 / 3} k^{2 / 3}\left(k / k_{d} \rightarrow 0\right),
$$

and

$$
E(k)=1.19 \epsilon^{2 / 3} k^{-5 / 3}\left(k / k_{d} \rightarrow 0\right) .
$$

Hence the Kolmogorov constant $F(0)=1.19 \simeq 1.2$, as reported in Ref. 6.

In the dissipation subrange the viscous dissipation is important; the nonlinear integral equations (17) and (18) are so complicated that it is a formidable task to obtain their exact solution. We are forced to seek some approximate solution. For this reason some trial solution, which contains several undetermined parameters, is used to approximate the exact solution. Then the equation-error method of parameter estimation of control theory ${ }^{7}$ is applied to the determination of these parameters, i.e., we choose these parameters in such a way that the trial solution will satisfy the Eqs. (17) and (18) as well as possible. In principle the accuracy of the trial solution can be improved by increasing the number of the undetermined parameters, but the amount of computation will increase enormously. As a preliminary attempt, a trial form containing a single parameter is used for $\eta(k)$, and a trial form containing two parameters is used for $F(x)$.

From (9) and (22), the simplest approximate formula for $\eta(k)$ is

$$
\begin{aligned}
\eta(k) & =0.268 \epsilon^{1 / 3} k^{2 / 3}+v k^{2} \\
& =0.268 \epsilon^{1 / 3} k^{2 / 3}\left[1+3.73\left(k / k_{d}\right)^{4 / 3}\right] .
\end{aligned}
$$

As a straightforward improvement of (24), the trial form of $\eta(k)$ is

$$
\begin{aligned}
\eta(k)= & 0.268 \epsilon^{1 / 3} k^{2 / 3} \\
& \times\left[1+A\left(k / k_{d}\right)^{2 / 3}+3.73\left(k / k_{d}\right)^{4 / 3}\right] .
\end{aligned}
$$

Here $A$ is an undetermined parameter. The trial form of $F(x)$ is

$$
F(x)=F(0)\left(1+B x^{\beta}\right) \exp \left(-C x^{\eta}\right),
$$


with $F(0)=1.19$. Here $B$ and $C$ are two undetermined parameters. Three different cases of (26) are to be studied in our numerical computation

$$
\begin{aligned}
& F(x)=F(0)(1+B x) \exp (-C x), \\
& F(x)=F(0)\left(1+B x^{2 / 3}\right) \exp \left(-C x^{2}\right),
\end{aligned}
$$

and

$$
F(x)=F(0)\left(1+B x^{2 / 3}\right) \exp \left(-C x^{4 / 3}\right),
$$

corresponding to $(\beta, \gamma)=(1,1),(2 / 3,2)$, and $(2 / 3,4 / 3)$, respectively. Equation (27b) is equivalent to Saffman's formula for the energy spectrum of the dissipation subrange. ${ }^{8} \mathrm{Pao}$ 's formula (3) is the special case of (27c) when $B=0$ and $C=1.5 F(0)$.

By the definition of $\epsilon$, we have

$$
\epsilon=2 \int_{0}^{\infty} v k^{2} E(k) d k \text {. }
$$

Substituting (1) and (26) into (28), we obtain

$$
B \Gamma\left(\frac{4}{3 \gamma}+\frac{\beta}{\gamma}\right)+C^{\beta / \gamma} \Gamma\left(\frac{4}{3 \gamma}\right)-0.42 \gamma C^{4 / 3 \gamma+\beta / \gamma}=0 \text {, }
$$

which is a constraint on $B$ and $C$. Here $\Gamma$ is the Gamma function.

When the exact solution is approximated by (1), (25), and (26), the (square) equation error of the energy equation (17) at wavenumber $k_{i}$ is defined as

$$
D_{t}\left(k_{i}\right)=\left[T\left(k_{i}\right) / 2 v k_{i}^{2} E\left(k_{i}\right)-1\right]^{2},
$$

and the (square) equation error of the $\eta$ equation (18) at $k_{i}$ is defined as

$$
D_{g}\left(k_{i}\right)=\left[G\left(k_{i}\right) / \eta\left(k_{i}\right)-1\right]^{2} .
$$

For the exact solution the equation error, $(30)$ and (31) are zero. The mean (square) equation error over $n$ different wavenumbers $k_{i}(i=1,2,3, \ldots, n)$ is

$$
D=\frac{1}{2 n} \sum_{i=1}^{n}\left[D_{t}\left(k_{i}\right)+D_{g}\left(k_{i}\right)\right],
$$

which is a complicated function of the undetermined parameters $A, B$, and $C$ of the trial forms (25) and (26). The optimum estimation of $A, B$, and $C$ is obtained by minimizing the mean equation error (32) under the constraint (29). The corresponding minimum of $D$, denoted by $D_{\min }$, can be used as a measure of the goodness of the trial solution. We will use "good," "poor," "better," and "worse" to describe the trial solution; it is not proper to say that the trial solution is "right" or "wrong." Of course the "best" solution is the exact solution. Unfortunately, we do not know how to obtain the exact solution.

\section{NUMERICAL RESULTS}

The $k_{i}$ in (32) are $n$ characteristic wavenumbers in the interesting (dissipation) range. Contrary to the parameterestimation problem of a stochastic process, the Eqs. (17) and (18) and their solution are deterministic. It is not of much benefit to let $2 n$ in (32) to be much greater than the number of the independent undetermined parameters. The turbulence experiments ${ }^{2,3,9-16}$ show that the most dissipation occurs in the range $k<0.5 k_{d}$, and the maximum of the one-dimen- sional dissipation spectrum locates between $0.05 k_{d}$ and $0.13 k_{d}$. Therefore we choose $n=2$ and

$$
k_{1}=0.05 k_{d}, \quad k_{2}=0.5 k_{d} .
$$

In our numerical computation the units are chosen in such a way that $\nu=1, \epsilon=1$, and therefore $k_{d}=1$. By using Eqs. (25), (27), and (30)-(32), the mean equation-error $D$ is numerically calculated for numerous different sets of parameters $A$, $B$, and $C$ under the constraint (29). Then, by comparison and interpolation, the optimum $A, B$, and $C$, which minimize the $D$, are determined. The resulting optimum $B$ and $C$ for (27) and the corresponding $D_{\min }$ are given in Table I.

According to Table I, Eqs. (27a) $-(27 \mathrm{c})$ are much better than Pao's formula (3), and the best of them is (27c). Hence

$$
F(x)=1.19\left(1+5.3 x^{2 / 3}\right) \exp \left(-5.4 x^{4 / 3}\right) .
$$

Its diagram is given in Fig. 1 together with Pao's result. ${ }^{5}$

\section{COMPARISON WITH EXPERIMENTAL RESULTS}

Experimental measurements are made of the one-dimensional energy spectrum $\varphi(k))^{2,3,9-16}$ The relationship between $\phi(k)$ and $E(k)$ is $^{2}$

$$
\varphi(k)=\int_{k}^{\infty}\left(1-\frac{k^{2}}{p^{2}}\right) \frac{E(p)}{p} d p,
$$

where

$$
\int_{0}^{\infty} \varphi(k) d k=\left\langle u_{1}^{2}\right\rangle,
$$

and

$$
\int_{0}^{\infty} E(k) d k=\frac{1}{2}\left\langle u_{1}^{2}+u_{2}^{2}+u_{3}^{2}\right\rangle .
$$

Here $u_{1}, u_{2}$, and $u_{3}$ are the three components of the random velocity. The normalized one-dimensional spectrum is defined as

$$
\tilde{\varphi}\left(k / k_{d}\right)=\varphi(k) /\left(\epsilon v^{5}\right)^{1 / 4} .
$$

From (1), (35), and (38),

$$
\tilde{\varphi}(y)=\int_{y}^{\infty}\left(1-\frac{y^{2}}{x^{2}}\right) x^{-8 / 3} F(x) d x .
$$

In terms of (34) and (39) the normalized one-dimensional spectrum is numerically calculated and given in Table II. Its diagram is given in Fig. 2 together with the experimental data of Refs. 9 and 10 and Pao's result. The corresponding normalized one-dimensional dissipation spectrum $\left(k / k_{d}\right)^{2}$ $\times \tilde{\varphi}\left(k / k_{d}\right)$ is also calculated numerically and given in Table II. Its diagram is given in Fig. 3 together with several sets of experimental data and Pao's result. The two dotted lines in Fig. 3 are, respectively, the upper and lower limits of experimental data of Grant et al., Pond et al., and Gibson; neither

TABLE I. Optimum estimation of $B$ and $C$.

\begin{tabular}{lcll}
\hline \hline Form of $F(x)$ & $B$ & $C$ & $D_{\text {min }}$ \\
\hline Eq. (27a) & 19.4 & 6.1 & 0.21 \\
Eq. (27b) & 2.8 & 6.3 & 0.14 \\
Eq. (27c) & 5.3 & 5.4 & 0.13 \\
Eq. (3) & 0 & $1.5 F(0)$ & 0.72 \\
\hline
\end{tabular}




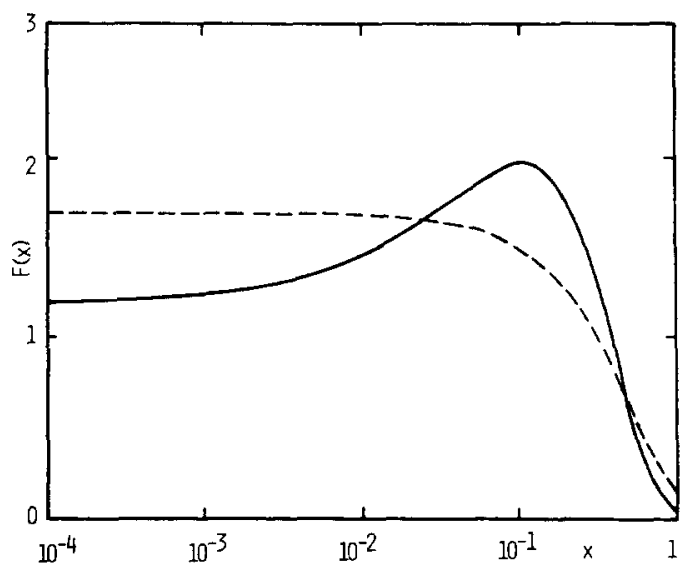

FIG. 1. $F(x)$ vs $x$. - present theoretical result; - - Pao's result.

of them represents one set of experimental data.

It is evident from Figs. 2 and 3 that our theoretical results are in good agreement with the experiments. The wavenumber $k_{\max }$, at which the theoretical dissipation-spectrum curve of Fig. 3 has a maximum, is $0.082 k_{d}$. The experimental values of $k_{d}$ scatter between $0.05 k_{d}$ and $0.13 k_{d}$.

Pao's result of Figs. 1-3 corresponds to the Kolmogorov constant $F(0)=1.7$, which makes Pao's formula (3) fit the experimental data of Grant et al. In order to make Pao's formula (3) fit the experimental data of Sheih et al., ${ }^{13} F(0)$ has to be 2.0. Essentially Pao's result is of semi-empirical nature.

\section{DISCUSSION}

Contrary to Pao's formula (3), (34) indicates that $F(x)$ is not a monotonically decreasing function. It increases slowly from 1.2 (when $x<10^{-4}$ ) to 2.0 (when $x \simeq 0.1$ ), then de-

TABLE II. Normalized one-dimensional spectrum.

\begin{tabular}{|c|c|c|}
\hline$k / k_{d}$ & $\tilde{\varphi}\left(k / k_{d}\right)$ & $\left(k / k_{d}\right)^{2} \tilde{\varphi}\left(k / k_{d}\right)$ \\
\hline $1.0 \times 10^{-4}$ & $1.85 \times 10^{6}$ & $1.85 \times 10^{-2}$ \\
\hline $2.0 \times 10^{-4}$ & $5.90 \times 10^{5}$ & $2.36 \times 10^{-2}$ \\
\hline $4.0 \times 10^{-4}$ & $1.90 \times 10^{5}$ & $3.03 \times 10^{-2}$ \\
\hline $6.0 \times 10^{-4}$ & $9.80 \times 10^{4}$ & $3.53 \times 10^{-2}$ \\
\hline $8.0 \times 10^{-4}$ & $6.15 \times 10^{4}$ & $3.94 \times 10^{-2}$ \\
\hline $1.0 \times 10^{-3}$ & $4.29 \times 10^{4}$ & $4.29 \times 10^{-2}$ \\
\hline $2.0 \times 10^{-3}$ & $1.42 \times 10^{4}$ & $5.68 \times 10^{-2}$ \\
\hline $4.0 \times 10^{-3}$ & $4.78 \times 10^{3}$ & $7.65 \times 10^{-2}$ \\
\hline $6.0 \times 10^{-3}$ & $2.55 \times 10^{3}$ & $9.16 \times 10^{-2}$ \\
\hline $8.0 \times 10^{-3}$ & $1.63 \times 10^{3}$ & 0.105 \\
\hline $1.0 \times 10^{-2}$ & $1.16 \times 10^{3}$ & 0.116 \\
\hline $2.0 \times 10^{-2}$ & $3.94 \times 10^{2}$ & 0.158 \\
\hline $4.0 \times 10^{-2}$ & $1.28 \times 10^{2}$ & 0.204 \\
\hline $6.0 \times 10^{-2}$ & $6.24 \times 10^{1}$ & 0.225 \\
\hline $8.0 \times 10^{-2}$ & $3.60 \times 10^{1}$ & 0.231 \\
\hline 0.1 & $2.28 \times 10^{1}$ & 0.228 \\
\hline 0.2 & 4.06 & 0.163 \\
\hline 0.3 & 1.07 & $9.59 \times 10^{-2}$ \\
\hline 0.4 & 0.325 & $5.19 \times 10^{-2}$ \\
\hline 0.5 & 0.107 & $2.66 \times 10^{-2}$ \\
\hline 0.6 & $3.65 \times 10^{-2}$ & $1.32 \times 10^{-2}$ \\
\hline 0.7 & $1.28 \times 10^{-2}$ & $6.29 \times 10^{-3}$ \\
\hline 0.8 & $4.58 \times 10^{-3}$ & $2.93 \times 10^{-3}$ \\
\hline 0.9 & $1.65 \times 10^{-3}$ & $1.33 \times 10^{-3}$ \\
\hline 1.0 & $5.94 \times 10^{-4}$ & $5.94 \times 10^{-4}$ \\
\hline
\end{tabular}

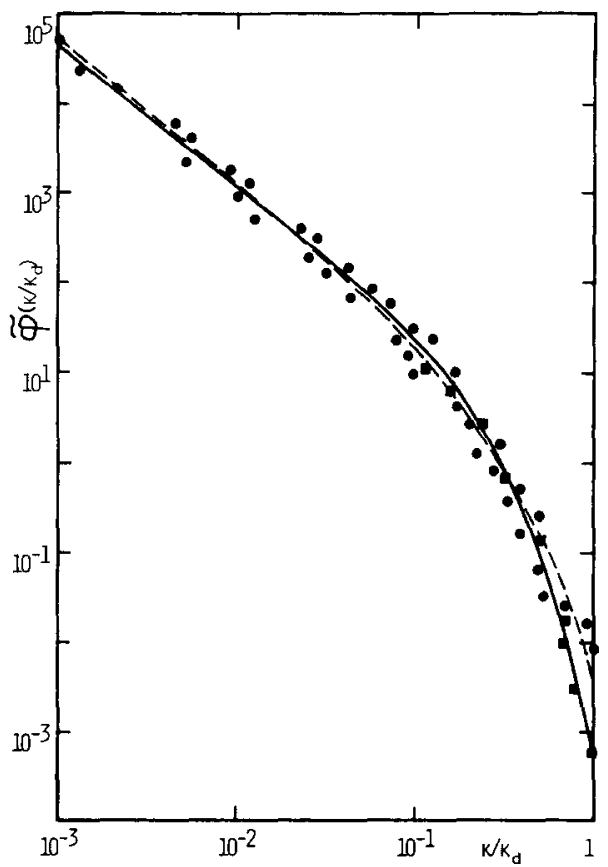

FIG. 2. The normalized one-dimensional energy spectrum. - present theoretical result; - - - Pao's result; $\square$ Gibson and Schwarz; Grant, Stewart, and Moilliet.

creases rapidly (see Fig. 1). The transition from the inertial subrange to the dissipation subrange is quite slow, occurring over a wide range of $k$. This is because the viscous dissipation approaches zero slowly as $k / k_{d}$ approaches zero.

In most turbulence experiments ${ }^{2,3}$ it is assumed that the $k^{-5 / 3}$ law is applicable when $k<0.1 k_{d}$. The experimental Kolmogorov constant is obtained by fitting a straight line of slope $-5 / 3$ to the experimental data for $\varphi(k)$ on a $\log -\log$ plot over some finite range of $k\left(k<0.1 k_{d}\right)$. According to Fig. 1 , when $k<0.1 k_{d}, F\left(k / k_{d}\right)$ is a slowly varying function of $k$, soby (1) and (35) $E(k)$ and $\varphi(k)$ will be approximately proportional to $k^{-5 / 3}$ over some finite range of $k$. It is interesting to note that if we fit a straight line of slope $-5 / 3$ to the theoretical spectrum curve of Fig. 2 for the interval $10^{-3}$

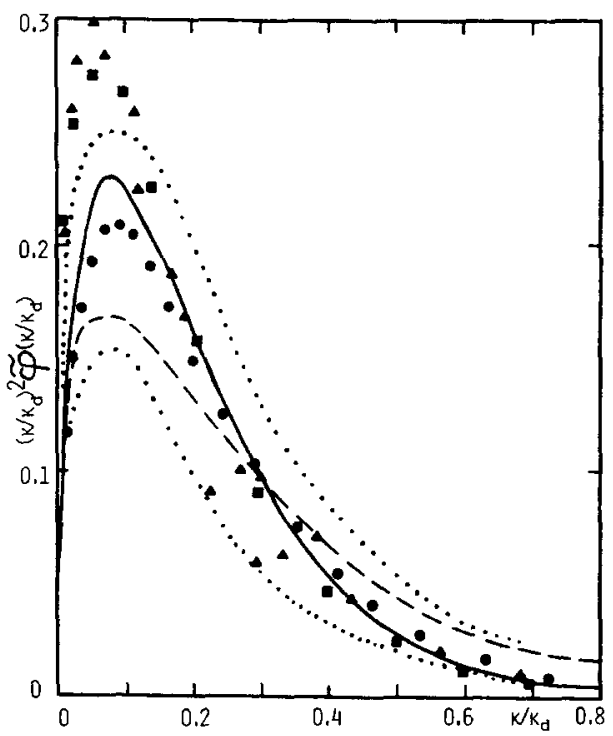

FIG. 3. The normalized one-dimensional dissipation spectrum. - present theoretical result; - - Pao's result; : : :Grant et al., Pond et al., and Gibson; Wyngaard; Champagne; $\Delta$ Sheih, Tennekes, and Lumley. 
$<k / k_{d}<0.1$, the resulting "Kolmogorov constant" is between 1.4 and 1.7 instead of $F(0) \simeq 1.2$. Hence in most turbulence experiments, instead of $F(0)$, we obtain some kind of experimental Kolmogorov constant which is greater than the theoretical Kolmogorov constant $F(0)$ and depends upon the range of $k / k_{d}$. This may be one of the reasons that the experimental values of the Kolmogorov constant scatter between 1.2 and 2 .

In some theories of turbulence the formal perturbation expansion in powers of the reciprocal of the Reynolds number $(\operatorname{Re})^{-1}$ is used. ${ }^{17}$ A perturbation method with $(\operatorname{Re})^{-1}$ as the small perturbation parameter cannot be applied to the study of the dissipation range, because the corresponding $(\mathrm{Re})^{-1}$ is not small. The probability distribution (4) is a perturbation solution of the Liouville equation of turbulence, ${ }^{6}$ but the corresponding small perturbation parameter is not $(\mathrm{Re})^{-1}$, and the distribution can be applied to the study of the dissipation subrange as well as the inertial subrange. The small perturbation parameter used in our theory ${ }^{6}$ is the distance between the Liouville operator and the FokkerPlanck operator in the functional space of operators with some properly defined measure. This distance is made as small as possible by adjusting an infinite number of parameters $\eta_{i}$ or $\zeta_{i}$, so it can be used as a small perturbation parameter. The agreement of our theoretical results with the experiments is the further justification of our theory.

\section{ACKNOWLEDGMENTS}

The author would like to express his appreciation to Professor C. M. Tchen of The City College of New York for his guidance and encouragement. The author is grateful to Dean Professor David H. Cheng of The School of Engineering of The City College of New York for his support.

\footnotetext{
${ }^{1}$ A. N. Kolmogorov, C. R. Dokl. Acad. Sci. URSS 30, 301 (1941).

'J. O. Hinze, Turbulence, 2nd ed. (McGraw-Hill, New York, 1975).

${ }^{3}$ A. Monin and A. M. Yaglom, Statistical Fluid Mechanics of Turbulence (MIT, London, 1975).

${ }^{4}$ W. Heisenberg, Z. Physik 124, 628 (1948).

${ }^{5}$ Y. H. Pao, Phys. Fluids 8, 1063 (1965).

${ }^{6} \mathrm{~J}$. Qian, Phys. Fluids 26, 2098 (1983).

${ }^{7}$ J. M. Mendal, Discrete Technique of Parameter Estimation (MarcelDekker, New York, 1973).

${ }^{8}$ P. G. Saffman, J. Fluid Mech. 16, 545 (1963).

${ }^{9}$ C. H. Gibson and W. H. Schwarz, J. Fluid Mech. 16, 365 (1963).

${ }^{10}$ H. L. Grant, R. W. Stewart, and A. Moilliet, J. Fluid Mech. 12, 241 (1962).

"S. Pond, R. W. Stewart, and R. W. Burling, J. Atmos. Sci. 20, 321 (1963).

${ }^{12}$ M. M. Gibson, J. Fluid Mech. 15, 161 (1963).

${ }^{13}$ C. M. Sheih, H. Tennekes, and J. L. Lumley, Phys. Fluids 14, 201 (1971).

${ }^{14}$ Noel E. J. Boston and R. W. Burling, J. Fluid Mech. 55, 473 (1972).

${ }^{15}$ F. H. Champagne, J. Fluid Mech. 86, 67 (1978).

${ }^{16}$ J. C. Wyngaard, Ph.D. thesis, Pennsylvania State University, 1967.

${ }^{17}$ S. A. Orszag, in Fluid Dynamics, edited by R. Balian and J. L. Peube (Gordon and Breach, New York, 1977).
} 